Voix et Images

voixetimages

\title{
Les États généraux du Théâtre professionnel au Québec (n'en faisons pas un drame)
}

\section{Bernard Andrès}

Volume 7, numéro 3, printemps 1982

Anne Hébert

URI : https://id.erudit.org/iderudit/200356ar

DOI : https://doi.org/10.7202/200356ar

Aller au sommaire du numéro

Éditeur(s)

Les Presses de l'Université du Québec

ISSN

0318-9201 (imprimé)

1705-933X (numérique)

Découvrir la revue

Citer cet article

Andrès, B. (1982). Les États généraux du Théâtre professionnel au Québec (n'en faisons pas un drame). Voix et Images, 7(3), 605-608.

https://doi.org/10.7202/200356ar d'utilisation que vous pouvez consulter en ligne.

https://apropos.erudit.org/fr/usagers/politique-dutilisation/ 


\title{
Les États généraux du Théâtre professionnel au Québec (n'en faisons pas un drame)
}

\author{
par Bernard Andrès, Université du Québec à Montréal
}

Les 7, 8 et 9 novembre 1981, se tenaient à Montréal les États généraux du Théâtre professionnel au Québec. Première manifestation du genre dans le milieu théâtral, cette assemblée des praticien(ne)s de tous àges (elle n'était pas réservée au "Jeune" théâtrel, aura permis à tout un secteur de l'activité culturelle de se situer par rapport à l'État, au public et à lui-même. Nul doute que la majorité des propositions votées à la plénière du 9 novembre concernait d'abord les rapports entre le théâtre et les divers paliers gouvernementaux. dans le cadre des problèmes de financement et souvent de survie de la plupart de ces organismes. Le style même des propositions témoignait de cette préoccupation fondamentale: s'assurer de l'appui inconditionnel de l'État pour aplanir l'ensemble des difficultés rencontrées par le milieu dans l'exercice de ses "missions socio-culturelles" (expression des États généraux). N'ayant guère le laisir de couvrir ici l'ensemble des 80 propositions (toutes ne purent d'ailleurs être soumises au vote), je ne m'attarderai pas sur les blocs "Régınalisation», "Lieux théâtraux" et "Statuts de l'artisan(e)". Mes observations porteront plutôt sur les autres propositions qui nous éclairent sur le type de rapport que le milieu entretient consciemment ou non avec le Pouvoir (fédéral, provincial ou municipal), et sur la facon dont il se situe dans le champ culturel québécois. Par-dela le succès ou l'échec des États généraux (point de vue auquel s'est souvent limitée la presse'). c'est tout le problème de la remise en question d'un appareil culturel en mal d'institutionnalisation. Appareil. le théâtre québécois l'est sans doute, si l'on s'en tient aux seuls organismes subventionnés: une centaine œuvrant sur une base régulière. Ils regroupent un millier d'artisan(e)s et drainent quelque 7 millions de dollars de subventions (dont près de $70 \%$ pour les onze compagnies dites institutionnelles). Institution, il cherche à le devenir, tant du côté des * grands * théâtres qui ne rejettent pas l'appellation, que du côté du "jeune" théâtre qui, faute de reconnaissance des pouvoirs publics, bénéficie de celle de l'École et de l'Unıversité (l'une qui le nourrit, souvent piteusement, et l'autre qui s'en nourrit, en matière de corpus d'étude, de sujets de revue, de thèses, etc.). Sans compter avec ces Ėtats généraux où d'aucuns ont voulu voir une sorte d'auto-légitimisation du “ jeune» théátre (j'y reviendrai). Et l'État là-dedans? 
Apparaissant dans la presque totalité des propositions votées, le terme «État semble effectivement symboliser l'actant principal et quasi mythique de cette entreprise de salut public, véritable épreuve de force lancée par les artisans du théâtre pour se tirer du pétrin administratif et financier (où baignent aussi bien et souvent plus dramatiquement les autres secteurs culturels: cinéma, peinture, littérature, etc.). Tour à tour et parfois simultanément opposant et adjuvant, l'État s'y trouve interpelé, qu'il s'agisse pour lui d'adopter une «politique culturelle globale et cohérente», en appliquant « les résolutions adoptées par les Ėtats généraux», ou de “ faire immédiatement du théâtre sa priorité culturelle en établissant un plan triennal afin de créer les conditions viables et adéquates pour l'exercice des différents métiers théâtraux». Tout se passe dans le milieu théâtral comme si cette tentative louable de se retrouver et de se prendre en main, ne pouvait se réaliser que sur le dos d'un tiers dont la fonction mobilisatrice - ou le rôle de bouc-émissaire - ne font aucun doute. L'ordre mème des blocs de propositions (reflétant celui des ateliers) est assez éloquent: avant mème de définir les objectifs et les statuts des membres, la charte du regroupement, il est question des «Politiques culturelles de l'État " (ce que le Pouvoir devrait faire ou empêcher. promouvoir ou interdire):

- plan triennal avec rapports d'étape:

- doublement et indexation du budget théâtral aux Affaires culturelles qui auraient $1 \%$ du budget provincial:

- affectation d'au moins $0.1 \%$ du budget fédéral au Conseil des Arts et de $1 \%$ du municipal au culturel:

- création de budgets pour le théâtre en milieu scolaire;

- promotion du théâtre québécois (au moins $50 \%$ des sommes):

- suppression des taxes d'amusement (ou réutilisation pour les lieux théâtraux) et de la censure municipale;

- abandon du critère de rentabilité dans l'évaluation des théâtres à but non lucratif:

- suppression des droits d'auteurs pour la traduction (en québécois) des pièces étrangères:

- création d'un Conseil des Arts du Québec, autonome et apolitique:

- reconnaissance et appui accru à la régionalisation ( Montréal est une région $\cdots$ ) et décentralisation (discussions serrées sur ce point);

- adoption de nouvelles catégorisations pour fin de subventions (du plus ponctuel au plus continu des fonctionnements: "Projets" - "Jeunes organismes" - "Théâtres accrédités" - "Théâtres agréés" - "Théâtres établis" ":

- etc...

Il est vrai qu'avec cette dernière résolution, le milieu théâtral tente réellement de se définir, même en termes de fonctionnement interne. On peut y voir à l'aube des années 80 et postérieurement à celui des Lettres, une forme d'institutionnalisation de ce champ culturel: lassés de se faire évaluer et cataloguer par les seuls fonctionnaires. les praticiens entendent 
imposer leurs propres étiquettes. II reste que ces nouveaux auto-collants recoupent passablement les anciens. Iis élargissent tout au plus le champ de légitimation à des pratiques jadis moins consacrées. Ainsi, la 3e catégorie ( $\alpha$ Théâtre accrédité ) englobe aussi bien des organismes à «fonctionnement ponctuel». Mais la limite avec la catégorie « Jeunes organismes» est des plus souples (ou des plus lâches): eux aussi peuvent avoir un fonctionnement continu. II va de soi que le cadre de ces États généraux et leur dynamique interne (consacrer un rassemblement ${ }^{2}$ ) interdisaient d'établir le consensus sur une classification plus nuancée ${ }^{3}$. Néanmoins la tentative était louable, tout comme celle destinée à forcer les praticiens à se brancher une bonne fois pour toutes sur le type de théâtre qu'ils entendaient de part et d'autre promouvoir. C'est là que le bât blesse et que les plus fortes divergences se sont manifestées... au point d'amener le rejet des propositions sur les "missions socio-culturelles». Désir de sauvegarder sa liberté d'action. refus de tout messianisme (mème de gauche), ou crainte pour certains d'avoir à reconnaître l'absence de perspective critique de leur théâtre? À ce propos, contrairement au point de vue officiel de l'ADT sur la prétendue philosophie des documents préparatoires qui auraient méconnu ou exclu l'optique des théâtres institutionnels, il semble que la grille proposée au vote intégrait bien toutes les orientations et combinaisons théâtrales possibles, en fonction de:

1) la finalité artistique (animation, expérimentation, exploration, intervention, tradition):

2) la nature de la programmation (création, étranger, national):

3) le médium choisi (acteurs, clowns, marionnettes, mimes);

4) le public souhaité (adultes et/ou enfants).

II est évident que, mème sans parler de priorité accordée aux quatre premières finalités au détriment de la dernière ( Tradition $\%)$, la simple mention d'une égalité d'importance entre celle-ci et celles-là, ne pouvait que provoquer la méfiance de ce qu'il faut bien appeler la génération des pionniers. Ces derniers (et premiers en date) étaient assez nombreux dans la salle pour faire pencher le vote en leur faveur. Ceci réduit à néant l'allégation de l'association à laquelle ils sont pour la plupart affiliés en tant que directeurs de théâtre: que dire de la volte-face du Conseil d'administration de I'ADT qui le soir même de l'ouverture, claquait la porte en invalidant ainsi tout le travail préparatoire du comité d'organisation (près de deux ans de bénévolat). Ce geste éminemment théâtral était posé sous le curieux prétexte d'un sentiment d'exclusion (mais I'ADT n'avait-elle pas participé activement depuis plusieurs mois au comité organisateur: on sait tout le travail qu'y investit son secrétaire avec les sept autres membres)... Pas question ici de décerner blâmes ou louanges ${ }^{4}$, mais d'observer que cette association avait eu le temps voulu pour exiger le droit de présence et de vote à ses mémbres (ainsi qu'aux relationnistes et aux directeurs de production). Aurait-elle voulu invalider 
l'entreprise qu'elle ne s'y serait pas pris autrement. II reste que le Pouvoir aura beau jeu à présent de mettre en doute la crédibilité de ces États généraux et de leur principale émanation: le Conseil Québécois du Théâtre. Mais ce dernier, élu dans les formes, est bien conscient des enjeux réels, des divergences et des points de ralliement d'un milieu qu'il représente clairement. ne serait-ce que sur la base des quelque 400 artisan(e)s venu(e)s se prononcer aux Etats.

Que les fonctionnaires se le tiennent pour dit, même si eux aussi ont pu se sentir frustrés par le huis-clos qui excluait aussi bien l'instance critique (à laquelle je me trouve moi-même rattaché). Et que dịre du public? Ne jouons donc pas les prima donna de coulisse et voyons le bon côté des choses. Non, les Etats généraux n'ont pas accouché d'une a nouvelle association du. Jeune théâtre»5. D'une façon ou d'une autre (même par l'absence, comme pour le théâtre d'Aujourd'hui), ils auront permis à tous et à chacun de se situer dans le champ théâtral. Là réside le succès d'une entreprise qu'on ne saurait bouder sans crainte du ridicule. Bravo, gens de la scène! Et vous, nous, gens de l'ombre, des coulisses et des chroniques, ne jouons pas la comédie du dépit (plus ou moins amoureux). Nous ne serons jamais aussi bons que les comédiens sur ce plan. Les Ėtats généraux se sont passés de nous? Pour l'amour du Théâtre, n'en faisons pas un drame $! . .$.

1. La presse a surtout couvert l'événement en termes de succès ou d'échec (surtout d'échecl, comme s'il s'était agi là encore d'un show à grande échelle monté dans la salle du Port-Royal...

2. La citation qui ponctue le texte de présentation du comité organisateur est assez éloquent (et savoureux) à ce propos. II cite Marilyn Fergusson (in Les enfants du Verseau, pour un nouveau paradigme): « (...) Le principe révolutionnaire introduit par Gandhi résoud le paradoxe de la liberté. ll l'a nommé Satyagraha, « force de l'âme* ou "force de la vérité», Satyagraha (...) signifie: "Ensemble nous pouvons résoudre le problème. C'est lui l'adversaire et non pas toi et moi».*

3. Pour une classification plus exhaustive, voir celle de Jèu n० 12 (été 79 ). Se référer également a l'essai de catégorisation le plus intéressant (surtout à propos du Jeune théâtre), celui récemment paru d'Adrien Gruslin, qui tient compte de toutes les composantes des pratiques théatrales : "Statut et fonctionnement", "Théàtre joué ", "Activités», «Lieux de diffusion", "Public visé, "Orientations" et "Mode de subvention.

4. Les lignes précédentes ne ménageaient pas certains ateliers dans leur vision quelque peu naive de l'Etat-Providence..

5. Sur le conflit des générations sous-jacent à ces États généraux, voir l'amusante formule de Gilbert David: «Ainsi pogné entre un Popa qui hayit ses Enfants et des enfants qui sont rongés par leur culpabilité cedipienne, le bonhomme Théâtre est maintenant assis entre deux chaises: celle des États trop généreux et celle de l'État tout court (Jeu $\left.n^{\circ} 21,1981-4\right)$. A noter que Jeu proposera une analyse détaillée de ces Etats généraux, dans le n०22. 